Relations industrielles

Industrial Relations

Managerial Motivation and Compensation, by H.L. Tosi, R.J. House and M.D. Dunette, (ed.), A Selection of Readings, East Lansing, Michigan, MSU Business Studies, 1972, 539 p.

\title{
Jean-Pierre Beaulieu
}

Volume 29, numéro 3, 1974

URI : https://id.erudit.org/iderudit/028540ar

DOI : https://doi.org/10.7202/028540ar

Aller au sommaire du numéro

\section{Éditeur(s)}

Département des relations industrielles de l'Université Laval

ISSN

0034-379X (imprimé)

1703-8138 (numérique)

Découvrir la revue

Citer ce compte rendu

Beaulieu, J.-P. (1974). Compte rendu de [Managerial Motivation and

Compensation, by H.L. Tosi, R.J. House and M.D. Dunette, (ed.), A Selection of

Readings, East Lansing, Michigan, MSU Business Studies, 1972, 539 p.] Relations

industrielles / Industrial Relations, 29(3), 622-623.

https://doi.org/10.7202/028540ar

Tous droits réservés (C) Département des relations industrielles de l'Université Laval, 1974
Ce document est protégé par la loi sur le droit d'auteur. L'utilisation des services d'Érudit (y compris la reproduction) est assujettie à sa politique d'utilisation que vous pouvez consulter en ligne.

https://apropos.erudit.org/fr/usagers/politique-dutilisation/ 
qu'autant ils n'ont pas de scrupules à se plier à des ordres venant de Moscou autant ils sont opposés à toute influence venant des Etats-Unis. La direction du CTC est amenée à lutter sur deux fronts.

On y constate le rôle important qu'ont joué certains personnages comme Charles Millard et surtout Pat Conroy. D'une manière générale, les individus dans leurs luttes pour le pouvoir ou pour le triomphe de leur façon de voir le syndicalisme, son rôle, son action et ses rapports avec les partis politiques apparaissent sous une lumière bien différente de celle que l'on trouve soit dans les journaux ou la propagande syndicale. En dévoilant les côtés mesquins qui se mêlent à l'action quotidienne et aux stratégies utilisées, l'auteur, dans son rôle d'observateur impartial effectue un travail de démystification sans pour cela diminuer les hommes ou le mouvement syndical.

On pourra ne pas être d'accord avec l'auteur dans toutes ses conclusions particulièrement sur le rôle positif qu'auraient joué les leaders communistes si on ne les avait pas délogés et sur son pessimisme vis-à-vis de la canadianisation des syndicats. Son ouvrage est toutefois une des contributions les plus valables à l'histoire du syndicalisme au Canada.

\section{Université Laval}

Les relations du travail, employeurs, personnel, syndicats, Etat, par Dimitri Weiss, Paris, Série "gestion sociale 》, Editions Dunod, 1974, 160 pp.

Ce nouveau livre que Dimitri Weiss consacre aux relations industrielles est plus que le précédent (que nous avions eu le plaisir de recenser dans Relations Industrielles, vol. 28, no 3) axé sur le système français des relations du travail. Par ailleurs, il comporte, comme l'ouvrage précédent, un très utile souci de clarification sur le plan des conceptsclés. Pour ne citer qu'un exemple, nous avons trouvé fort utiles les définitions et explications que l'auteur fournit au sujet des différents types de conflits du travail : de telles mises-en-ordre ne peuvent que faciliter l'identification des problèmes, puis leur discussion ordonnée, deux choses qui, en relations industrielles, sont éminemment utiles. Nous ne croyons pas nous aventurer trop loin en disant que pour le lecteur nord-américain, le chapitre de l'ouvrage qui traite de \& La Négociation Collective» (Chapitre 3) et celui qui traite des «.... Institutions de représentation du personnel dans l'entreprise » (Chapitre 4) revêtiront un intérêt particulier. D'une façon plus précise, on ne peut être, de ce côté-ci de l'Atlantique, qu'intéressé par l'examen et la discussion de questions et développements résultant de la coexistence, au sein d'entreprises françaises, du Comité d'Entreprise et de la Section Syndicale. Et même s'il est trop loin pour prendre position, le lecteur d'ici ne manquera pas de relever avec intérêt, au sujet de la convention d'entreprise, les commentaires qui sont offerts et cités dans l'ouvrage (voir le sous-chapitre intitulé «les conventions d'entreprise ou d'établissement » pp. 58-61). Pour ceux qui, parfois, sont friands de rapprochements, l'ouvrage contient aussi des citations qui ne manqueront pas, même à distance, de faire réfléchir. Par exemple, après avoir évoqué le «syndicalisme d'entreprise», on propose une distinction entre le syndicalisme d'entreprise » à l'américaine » et la "présence syndicale dans l'entreprise ", cette dernière expression étant présentée comme collant peut-être plus à la réalité française (p. 71). En laissant évidemment à l'auteur la responsabilité de ses orientations et de ses choix, l'on ne peut que conseiller la lecture de ce livre, clairement et vivement écrit. En plus de leur valeur incontestablement éducative, des ouvrages tels que celui-ci et traitant du système de relations de travail d'autres pays, nous aident à mieux situer, et à mieux comprendre les particularités de notre propre système.

Félix QUINET

Commission des relations de travail dans la fonction publique,

Ottawa

Managerial Motivation and Compensation, by H. L. Tosi, R. J. House and M. D. Dunnette, (ed.), A Selection of Readings, East Lansing, Michigan, MSU Business Studies, 1972, 539 p.

Présenter une série d'articles traitant de la motivation et de la rétribution, articles d'intérêt à la fois pour les administrateurs et les théoriciens du Management, tel était l'objectif des éditeurs de 
cet ouvrage. Devant la profusion d'articles déjà publiés sur ces sujets on pourrait penser qu'il n'avait que l'embarras $\mathrm{du}$ choix. Toutefois l'on sait que plusieurs de ces articles sont décevants par leur peu de rigueur.

C'est justement sur ce point que l'ouvrage de Tosi et ses collègues est valable. Ce volume est partiellement issu d'un séminaire commandité par la «McKinsey Foundation for Management Research » sur les sujets précités. Non seulement plusieurs des articles présentés sont inédits mais le regroupement qui est fait est des plus intéressant.

L'introduction présente une vue d'ensemble du sujet traité et plusieurs des articles qui suivent sont cités et situés les uns par rapport aux autres.

La seconde partie expose les théories de base fréquemment utilisées tant par les praticiens que par les chercheurs dans le domaine de la motivation et de la rétribution.

La partie suivante est particulièrement intéressante en ce qu'elle nous présente les critiques formulées à l'endroit des théories énoncées dans la section précédente.

La quatrième partie est à la fois plus empirique et expérimentale puisqu'elle livre les résultats d'une série d'études menées en laboratoire et sur le terrain.

La cinquième partie aborde la question d'évaluation du rendement, de notation des employés. Les articles contenus dans cette partie présentent des pratiques administratives reliées à la question du rendement et de la rétribution.

Enfin la dernière partie laisse voir que le sujet de la rétribution et de la motivation pose encore plusieurs questions auxquelles les théoriciens et chercheurs autant que les praticiens n'ont pas de réponses finales et satisfaisantes.

Somme toute c'est un volume qui malgré un certain goût de déjà vu, c'est la faiblesse de tous les ouvrages de ce genre, apporte une contribution valable dans le domaine des sciences appliquées du comportement. La diversité et la qualité des articles retenus et plus spécialement leur regroupement en diverses catégories qui se complètent avantageu- sement nous porte à croire que les éditeurs ont atteint leur objectif.

\section{Jean-Pierre BEAULIEU}

Alcan, Arvida

La protection du revenu dans les conventions collectives au Québec, par Jean Bernier avec la collaboration de Réal Allard et Pierre Dionne, Québec, Ministère du travail et de la maind'œuvre, 1974, 324 pp.

Le professeur Jean Bernier, avec la collaboration de Réal Allard et de Pierre Dionne, a incontestablement fait œuvre utile en nous présentant son étude sur «La protection du revenu dans les conventions collectives au Québec ». Il s'agit d'un ouvrage qui mérite une place de choix dans la littérature analytique en relations industrielles, car d'une façon systématique et fouillée, il nous fait mieux connaître et apprécier l'importante contribution apportée par la convention collective à l'édification de la Sécurité Sociale au Québec, contribution que l'on constate d'ailleurs aussi dans d'autres provinces canadiennes. Il s'agit donc d'une étude des dispositions de conventions collectives visant à la protection du revenu du travailleur dans des situations - ou, plus précisément, face à des risques que les auteurs ont clairement définis et différenciés. Le sujet de l'étude est, dès les premières pages, bien situé : "Dans la mesure où les régimes publics visaient à assurer un minimum de protection à tous, les parties contractantes aux conventions collectives demeuraient libres de conclure des ententes ayant pour objet de compléter les mesures d'ordre public en vue d'assurer une meilleure protection aux travailleurs régis par conventions collectives... Ce sont précisément ces régimes complémentaires de protection du revenu élaborés de façon contractuelle et prévus dans les conventions collectives qui font l'objet de la présente étude ». (p. 12).

Mentionnons aussi que la notion de " régime de protection du revenu », et de «risque » font l'objet de céfinitions et d'explications claires et d'ailleurs indispensables à la compréhension de l'étude (pp. 20 et suivantes). Il faut souligner également le soin que l'on a mis à décrire les principales caractéristiques de 